\title{
Risk Assessment and Control for Main Hazards in Reverse Osmosis Desalination Plants
}

\author{
Ahmed Fathy Awwad ${ }^{1}$, Mohammed Hassan Gobran ${ }^{2}$, Radwan Mostafa Kamal', \\ Mohammed Abdel Hameed Boraey ${ }^{2, *}$ \\ ${ }^{1}$ Health and Safety Department, Ittefaq Steel Company, Dammam, KSA \\ ${ }^{2}$ Mechanical Power Engineering Department, Zagzig University, Zagazig, Egypt

\section{Email address:} \\ hse.eng88@yahoo.com (A. F. Awwad),mhgobran@yahoo.com (M. H. Gobran), info@ecord1.com (R. M. Kamal), \\ maboraey@gmail.com (M. A. H. Boraey) \\ ${ }^{*}$ Corresponding author
}

\section{To cite this article:}

Ahmed Fathy Awwad, Mohammed Hassan Gobran, Radwan Mostafa Kamal, Mohammed Abdel Hameed Boraey. Risk Assessment and Control for Main Hazards in Reverse Osmosis Desalination Plants. Industrial Engineering. Vol. 2, No. 1, 2018, pp. 1-11. doi: $10.11648 /$ j.ie.20180201.11

Received: May 12, 2018; Accepted: May 29, 2018; Published: June 28, 2018

\begin{abstract}
As the manpower is the most valuable and important element for production in any industrial plant or organisation, so their health and safety must be managed to protect them against the reasonably foreseeable risks. In this paper risk assessment is carried out for five Reverse Osmosis desalination plants to detect the main hazards in such type of plants. Health safety executive -five steps to risk assessment model is used including $5 \times 5$ risk matrix to rank the risk level and then represented by color code to clarify the priority. The materials, equipment, working place and peoples included in the operation are considered while assessing the risk and working activities are monitored and analyzed. The most common hazards are found in working at height, working inside confined spaces or under water, exposure to noise, contacting with uncovered rotating equipment, electricity, high pressurized fluid and fire. The control measures are introduced to mitigate the level of identified risks to an acceptable level like developing a permit to work system and procedures for confined space entry or working under water, lock out and tag out of the power supply while dealing with electrical equipment, guarding all rotating parts of the machines, provide the right equipment for working at height, reduce the exposure time to noise and providing sufficient training, information and safety instructions to the employees. This paper can be used as a safety booklet for RO desalination plants operators.
\end{abstract}

Keywords: Risk Assessment, Hazard Identification, Occupational Health and Safety, Reverse Osmosis, Desalination Plants, Hazards Control Measures

\section{Introduction}

As generally known, water is the most essential element for life; unfortunately a little amount of water available in the earth can be used by human being as healthy water. [1] And even this amount of water is too little to cover the growing population in the world especially in high density areas. In these conditions and because of scariness of pure water sources, desalination of brackish and sea water comes as a key solution for water problem. [2] Several billion gallons of water are produced daily by desalination but still the needed amount of water is beyond the produced one, so more and more desalination plants are required. Reverse osmosis (RO) desalination is proved to be the most reliable, cost effective, and energy efficient in producing fresh water compared to other desalination technologies. It is the fastest-growing desalination technology with the greater number of installation around the world and produce around $75 \%$ of the total desalinated water. [3]

RO desalination plants are not used only for producing fresh water for drinking purpose, but also it is widely used for industrial purposes, many industries depend on pure water like iron \& steel industry, medicine industry, food industry... etc. so there are a numerous number of RO desalination plants around the world.

Due to the nature of work, complexity of workplace and 
difficulty of activities, workers in RO desalination plants are exposed to high range of hazards e.g. physical, biological, psychosocial, ergonomic, mechanical, and electrical and fire. Such complicated working places include high level of risks which should be assessed accurately in order to be considered while managing the safety and health of workers. [4]

Occupational health and safety is a major aspect that any industrial plant must manage proactively to secure the continuity of production and prevent accidents. Lack of occupational safety and health management has severe effect on plants as well as people such as, loss of competent manpower, production delay, complaints, increasing insurance cost, disciplinary actions, bad reputation, unsatisfied stakeholder and deterioration of business. Risk assessment is an essential tool for managing health and safety, identifying the hazards and controlling the risks.

Risk is a part of our life. Although we cannot avoid risks, we can take reasonable measures to control them effectively. There will be always a small residual risk in working activities but without this risk no activity can be done.

Risk assessment is a comprehensive examination of anything in the workplace that may lead to harm of workers. It helps to make sure that serious work place risk are managed by reasonably practicable control measures and allows the decision maker to prioritize the actions that should be taken.

Risk can be estimated by consideration of two factors the likelihood of the hazardous event to occur and the severity of consequence if the risk occurred, so the risk level can be calculated by multiplying the likelihood and consequence [5]

$$
\text { Risk Level }=\text { Likelihood } \times \text { Consequence }
$$

V. K. HANADA [6] discussed the main factors of accident in desalination plants; he depended on the analysis of operator's tasks and divided them into three parts, functional or activity development, activity identifications, and manpower assessment to prove that the application of human factor method and tools as integrated part of design process reduces the incident of early operability problems. ASEM [7] Research Task Force on Risk Based Inspection Guidelines (produces guidelines on the in-service, inspection, technique, frequency and scope based on the risk). ASEM report 1993 introduced evident that operator's action could significantly reduce the risk of accident to a plant and further risk of component failure and mitigate failure of control component. DONAGI [8] depended on Job Safety Analysis (JSA) to identify the main common hazards in water treatment plants through observing of the main tasks and used equipment; he categorized hazards into five categories, accident hazards, physical hazards, chemical hazards, biological hazards, and psychosocial hazards. A. M. Shams El Din [9] focused on the chemical hazards in water desalination plants, he discussed the main tasks that include dealing with chemicals and identified the chemical agents and its harm to operator's health or even its effect to the environment.

This paper aims to assess the main significant hazards relative to RO desalination plants, through carrying out safety tours, inspections and continues monitoring for work activities in five RO plants named NASCO, DRI, FSPC, MRM and ISPC, in order to be able to put hazards in order and introduce the necessary controls to reduce the risk to accepted level in order to guide concerned people while managing health and safety in such type of plants.

\section{Risk Assessment Approach \& Methodology}

The risk assessments will be carried out based on HSE five steps to risk assessment approach. Following is a brief description of that model. [5]

Five steps to risk assessment model:

This is one of the most simple and effective risk assessment models which was developed by Health Safety Executive (HSE), this model consists of five steps as follows:

Step one: Identify the hazard:

In this stage a walk around the work place to be carried out to detect what is going and look for anything that could be expected to cause harm taking into consideration the following elements

a) Location: to check the design of the workplace and ensure that all safety requirements were covered such as permanent aisle, emergency exit, lighting and ventilation.

b) Activities: to check for routine and non-routine tasks to ensure that all activities are done in a safe way like manual handling, lifting and transporting materials, working at height.

c) Equipment \& materials: to check for the tools, machines, substances that are used in the work place to ensure that they were installed safely and are running or used in the safe way based on the manufacturer's instructions.

d) Peoples: to observe all humans and their behavior inside the work place including workers, visitors and contractors to ensure that all safety aspects were considered such as staff training or contractor selecting criteria.

By doing so, almost all reasonably foreseeable hazards will be identified.

Step two: Estimate the risk:

In this stage risk is estimated to see how serious it is and be able to prioritize all risks and manage them in order as per their priority. To make the right estimation of the risk there are three factors that should be considered.

a) Who might be harmed and how: it is necessary to identify who might be harmed by this risk as the evaluation of the risk may vary based on the one or group who might be harmed e.g. the risk of knife is low when the targeted persons are adults and it is very high when amateurs are exposed to its risk.

b) How likely is that things could go wrong, as the right estimation depends on the likelihood e.g. the risk of 
tripping because of floor hole could be high if the hole in a crowded road while the same risk could be low if the hole in a rarely occupant road.

c) How severe would the outcomes be, as the risk rate depends on the severity of the consequence and the rate of the same risk definitely varies based on the severity e.g. the risk rate of fire in cheap goods store is low while the risk of fire in expensive materials store is high.

After considering these three factors the risk rate can be easily estimated by multiplying the likelihood and severity.

In order to make the risk estimation clear and simple, numbers can be used to represent the likelihood and severity. Five points scale can be used to express both likelihood and consequence and give good classification as in the below figure:

\begin{tabular}{|c|c|c|c|c|c|}
\hline likelihood & $\begin{array}{c}\text { In significant } \\
(1)\end{array}$ & Minor (2) & Moderate (3) & Major (4) & $\begin{array}{c}\text { Catastrophic } \\
(5)\end{array}$ \\
\hline Very likely (5) & 5 & 10 & 15 & 20 & 25 \\
\hline Likely (4) & 4 & 8 & 12 & 16 & 20 \\
\hline Fairly likely (3) & 3 & 6 & 9 & 12 & 15 \\
\hline Unlikely (2) & 2 & 4 & 6 & 8 & 10 \\
\hline $\begin{array}{c}\text { Very unlikely } \\
(1)\end{array}$ & 1 & 2 & 3 & 4 & 5 \\
\hline
\end{tabular}

Figure 1. Severity and consequences risk matrix.

From the previous scaling of likelihood and consequence, $5 \times 5$ risk matrix is obtained.

In addition to using numbers, colour codes can be good presentation and indication tools to classify risks as in figure 2 :

\begin{tabular}{|c|c|c|c|c|c|}
\hline Severity & $\begin{array}{c}\text { In significant } \\
\text { likelihood }\end{array}$ & Minor (2) & Moderate (3) & Major (4) & $\begin{array}{c}\text { Catastrophic } \\
(5)\end{array}$ \\
\hline Very likely (5) & 5 & 10 & 15 & 20 & 25 \\
\hline Likely (4) & 4 & 8 & 12 & 16 & 20 \\
\hline $\begin{array}{c}\text { Fairly likely } \\
(3)\end{array}$ & 3 & 6 & 9 & 12 & 15 \\
\hline $\begin{array}{c}\text { Unlikely (2) } \\
\text { Very unlikely } \\
(1)\end{array}$ & 2 & 4 & 6 & 8 & 10 \\
\hline
\end{tabular}

Figure 2. Colour coded risk matrix.

Now, each identified risk can be described by number and colour code which make it easy to prioritize all risks to be ready for evaluating each one to identify the suitable control measures:

Step three: evaluate the risk

After estimation the risk and determining its level, it's the time to see what should be done and which action should be taken and when. The action should be suitable to the risk level, it means that the higher the risk level is the more urgent and serious action is needed. The following table shows the suitable action for each risk level.

Table 1. Control action based on risk level.

\begin{tabular}{ll}
\hline Risk level & Action \\
\hline $20-25$ & Stop - stop the activity till immediate action is taken to control and reduce the risk \\
$15-16$ & Urgent action - take immediate and rigorous action without stopping the activity if practicable \\
$8-12$ & Action - take suitable action within specified time scale to improve the existing control to reduce the risk level \\
$3-6$ & Monitor - continuously monitor the existing control measures to ensure it still effective and improve if required \\
$1-2$ & No action - no further action is needed but the assessment should be reviewed periodically \\
\hline
\end{tabular}

Step four: Record findings

Any risk assessment must be recorded and shared with workers and concerned staff to improve the performance and ensure that the risk assessment is beneficial. Recording should consider some main points such as:

a) Date, time and details of assessor.

b) Details of the location, equipment and activities.

c) Hazards that were identified and its risk level.

d) Existing and additional required control measures.

Step five: review risk assessment.

Risk assessment needs to be reviewed periodically to ensure it is still valid and effective in addition to reviewing in case of changes e.g. new equipment, staff turnover, using of new materials or new information comes to the light.

\section{Risk Assessments of RO Desalination Plants Related Hazards}

Following are eight risk assessments for the most significant relative hazards in RO desalination plants. 


\subsection{Risk Assessment for Working at Height}

\subsubsection{Assessment Methodology}

This risk assessment will be semi quantitative which includes systematic identification and analysis of falling from height while doing maintenance inspection or cleaning work to check the efficiency of current control measures and decide if there is a need for improvement. It will follow 5 steps to risk assessment model and will use $5 \times 5$ risk matrix to represent the likelihood, consequence and risk level.

\subsubsection{Hazard Identification}

As RO desalination plants contain a lot of water or chemical tanks and filters with a considerable height $(\geq 3 \mathrm{~m})$ or medium height $(\geq 1.8 \mathrm{~m})$ but in a critical place e.g. beside sharp edges or chemical tanks which could lead to severe injuries in case of falling [10]. Usually workers need to do some activities above those tanks or filters such as monitoring the water level or changing filter cartridge.

Some of these tanks are opened (feed water tanks) which threaten the worker life if fall inside water and some tanks are covered but the roof is fragile which could collapse in case of heavy load e.g. group of workers or heavy equipment.

Falling from this height could cause fatalities or severe injuries.

\subsubsection{Estimate the Risk}

After Job Safety Analysis (JSA) for working at height activities, it was noted that the average time of working at height is $48-56$ hours per month for a team of 2-4 workers which is considered significant exposure time in addition to some circumstances that increase the likelihood of falling such as wet or slippery roof and high speed wind, so the likelihood is estimated as medium (3).
In case of falling the consequence will be severe either because of falling from height, falling in water or because of hitting by surrounding objects which could lead to massive injury or fatalities, so the consequence is estimated as very high (5)

Based on the likelihood and consequence estimation, the risk level is estimated as 15, which is considered high risk level with orange colour code.

\subsubsection{Evaluate the Risk}

As the risk rate is ranked by high, urgent action must be taken to protect employees and ensure they are safe while carrying out work at height.

Recommended actions:

a) Avoid working at height as possible such as depending on sensors to determine the water level inside tanks instead of visual monitoring.

b) Provide the right and suitable equipment for working at height such as man lift, which provide more protection and safety to workers than ladders.

c) Prevent lone working above tanks especially opened ones.

d) Provide fall arrest system and the required equipment such as full body harness, life lines and anchor points.

e) Provide training to workers about the risks included in such tasks, the safety precautions and how to use the fall arrest equipment.

f) Continuous monitoring and supervision to ensure compliance with the safety instructions and prevent shortcuts that done by workers.

By applying these control measures the risk rank is expected to be reduced from the high level with orange colour code to low level with light green colour code, which require keeping monitoring and improvement in case of any changes.

\begin{tabular}{|c|c|c|c|c|c|}
\hline $\begin{array}{c}\text { Severity } \\
\text { likelihood }\end{array}$ & $\begin{array}{c}\text { In significant } \\
(1)\end{array}$ & Minor (2) & Moderate (3) & Major (4) & $\begin{array}{c}\text { Catastrophic } \\
(5)\end{array}$ \\
\hline Very likely (5) & 5 & 10 & 15 & 20 & 25 \\
\hline Likely (4) & 4 & 8 & 12 & 16 & 20 \\
\hline Fairly likely (3) & 3 & 6 & 9 & 12 & Before(15) \\
\hline Unlikely (2) & 2 & After (4) & 6 & 8 & 10 \\
\hline Very unlikely (1) & 1 & 2 & 3 & 4 & 5 \\
\hline
\end{tabular}

Figure 3. Risk matrix for working at height.

\subsection{Risk Assessment for Working Inside Confined Space}

\subsubsection{Assessment Methodology}

This risk assessment will be qualitative because of the difficulty of estimating the time of working inside the confined space and expected number of workers. The assessment will follow five steps to risk assessment model depending on the likelihood of risk to occur and the severity of consequence in case of occurrence.

\subsubsection{Hazard Identification}

RO desalination plants contain a lot of confined spaces almost are tanks. Workers have to enter those confined spaces to carry out some tasks such as removing fouling, repairing leakage or sterilization.

Confined space entry could lead to many risks e.g. asphyxiation due to oxygen deficiency, toxicity because of presence of poisonous gases or accidental submerging by fluids.

\subsubsection{Estimating the Risk}

Confined space entry is not considered frequent or routine job, it is non-routine job and occurs every long and nonspecified period which makes the likelihood low (2), but in case of entry and something wrong happen, the consequence will be severe and almost reach to fatality and usually in confined space accidents the number of victims are more than one. For those reasons, the severity of consequence is 
estimated as very high (5).

Based on the likelihood and consequence estimation, the risk level is 10 , which is considered medium risk level with yellow colour code.

\subsubsection{Evaluate the Risk}

As the risk rate was estimated by 10 with yellow colour code, actions should be taken within a specified time scale to improve the safety standard and keep workers safe.

Recommended actions:

a) Reduce the need to enter confined spaces as possible e.g. adding chemicals to the water to prevent fouling formation to avoid the need of manual cleaning.

b) Develop and activate confined space entry permit system and strictly prevent anyone from entry except after getting permit from the authorized person.

c) Issue rigorous instruction to carry out gas test before entry to ensure that oxygen, poisonous gas and lower explosive limit are within the permissible limit.

d) Provide the required safety equipment such as selfcontained breathing apparatus and gas detectors in addition to provision of suitable training about usage, calibration and maintaining of this equipment.

e) Provide rescue plan in case of emergency e.g. how to get injured people out of the confined space and provide the medical aid to them.

f) Identify and list all confined spaces inside the plants, share these information with all workers and strictly monitor workers compliance with the safety instructions.

By applying these control measures the risk rank is expected to be reduced from the medium level with yellow colour code to very low level with dark green colour code, which requires no action but periodical review should be in place.

\begin{tabular}{|c|c|c|c|c|c|}
\hline $\begin{array}{c}\text { Severity } \\
\text { likelihood }\end{array}$ & In significant (1) & Minor (2) & Moderate (3) & Major (4) & Catastrophic (5) \\
\hline Very likely (5) & 5 & 10 & 15 & 20 & 25 \\
\hline Likely (4) & 4 & 8 & 12 & 16 & 20 \\
\hline Fairly likely (3) & 3 & 6 & 9 & 12 & 15 \\
\hline Unlikely (2) & 2 & 4 & 6 & 8 & Before(10) \\
\hline Very unlikely (1) & 1 & After (2) & 3 & 4 & 5 \\
\hline
\end{tabular}

Figure 4. Risk matrix for confined space.

\subsection{Risk Assessment for Noise}

\subsubsection{Assessment Methodology}

This risk assessment will combine between both qualitative and quantitative approaches through calculating the exposure time and noise level and comparing them with the standard. The assessment will follow five steps to risk assessment model.

\subsubsection{Hazard Identification}

While carrying out maintenance or periodical inspection in the high pressure pumps area, workers expose to significant level of noise which is produced by high pressure pumps. This was easily noted by applying the rough rule of thumb (the noise is so loud that person cannot reasonably hear normal conversation at a distance of two meters) which means that the noise level exceeds the permissible ones as shown in the below figure no 5 , based on control of noise at work regulation 2005. Exposure of operators in that area can be neglected as high pressure pumps are kept in separate compartment where there are no operators.

\subsubsection{Estimate the Risk}

After observation of the work schedule for preventive maintenance, inspection and approximate calculation of breakdown times, it was noted that two maintenance staff are exposed the noise in that area from three to four hours daily. The measurement of noise level in that area was found $94 \mathrm{db}$.
By comparing the exposure and the noise level with the standard [11] as in the below figure, it was found that the exposure limit highly exceeds the permissible one. Such exposure level could badly affect the hearing accuracy of employees over long term exposure and could cause hearing loss.

\begin{tabular}{|c|c|}
\hline Sound Level $\mathbf{d B}(\mathbf{A})$ & $\begin{array}{c}\text { Exposure Equivalent to } \\
\mathbf{8 5} \mathbf{d B}(\mathbf{A}) \mathrm{L}_{\mathrm{EP}, \mathrm{d}}\end{array}$ \\
\hline 85 & 8 hours \\
\hline 88 & 4 hours \\
\hline 91 & 2 hours \\
\hline 94 & 1 hour \\
\hline 97 & 30 mins \\
\hline 100 & 15 mins \\
\hline 103 & 7.5 mins \\
\hline
\end{tabular}

Figure 5. Permissible exposure limit to noise.

As the exposure time exceeds the permissible limit (safe limit) the likelihood of the harm to employees hearing is estimated as medium (3) and the consequence is estimated as high (4).

Based on the likelihood and consequence estimation, the risk level is estimated as 12 , which is considered medium risk level with yellow colour code.

\subsubsection{Evaluate the Risk}

As the risk rate is estimated by 12 with yellow colour code 
in the $5 \times 5$ risk matrix, actions should be taken within a specified time scale to improve the safety standard and keep workers safe.

Recommended actions:

a) The control actions will start by short term controls through applying safe system of work to reduce the exposure time by activating job rotation between the staff to ensure that no one will expose to that level of noise more than one hour daily in addition to providing suitable Personal protective equipment e.g. ear plugs.

b) Then long term actions should be taken by isolation of pumps in a separate booth which is made from sound absorptive materials with a suitable design as shown in figure 6 .

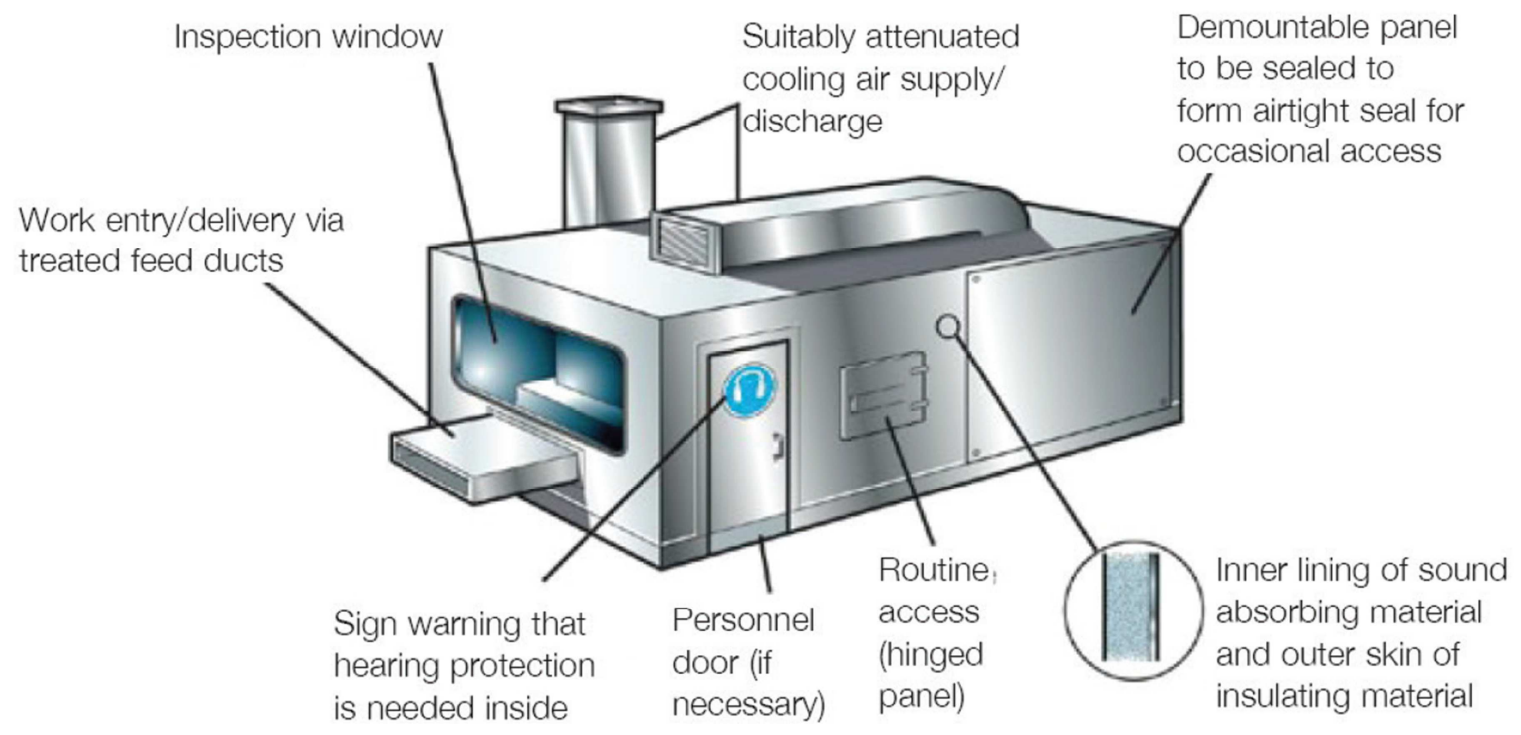

Figure 6. Sound resistant booth [12].

c) In addition to the previous actions, health surveillance should be carried out annually to the targeted employees through audiometry medical check.

By applying these control measures the risk rank is expected to be reduced from the medium level with yellow colour code to low level with light green colour code, which requires continuous monitoring to ensure that the existing control measures still valid and effective and that worker comply with the instruction.

\begin{tabular}{|c|c|c|c|c|c|}
\hline $\begin{array}{c}\text { Severity } \\
\text { likelihood }\end{array}$ & $\begin{array}{c}\text { In significant } \\
(1)\end{array}$ & Minor (2) & Moderate (3) & Major (4) & $\begin{array}{c}\text { Catastrophic } \\
(5)\end{array}$ \\
\hline Very likely (5) & 5 & 10 & 15 & 20 & 25 \\
\hline Likely (4) & 4 & 8 & 12 & 16 & 20 \\
\hline Fairly likely (3) & 3 & 6 & 9 & Before (12) & 15 \\
\hline Unlikely (2) & 2 & After (4) & 6 & 8 & 10 \\
\hline Very unlikely (1) & 1 & 2 & 3 & 4 & 5 \\
\hline
\end{tabular}

Figure 7. Risk matrix for exposure to noise.

\subsection{Risk Assessment of Fire}

\subsubsection{Assessment Methodology}

This assessment will be qualitative and will follow five steps to risk assessment model to identify the likelihood of fire to happen and the severity of consequence if fire occurred.

\subsubsection{Identify the Hazard}

As there is big amount of stored fuel (15 cubic meter of diesel) inside the plant, fire is considered as a significant potential risk which could lead to injuries, fatalities or damage to the work place. In addition to fuel there are other causes which could lead to fire e.g. hot works, faulty electric equipment and flammable chemicals.

\subsubsection{Estimate the Risk}

Based on the design of the plants and after review of the plants layout, the diesel tank is usually separate and far from any other part of the plant, and is kept inside a concrete tank as a secondary containment. The nearest part of the plant to the diesel tank is the generator compartment which is 2 meters far from the tank. Also it is not usual that workers or any other one need to work beside the diesel tank, these circumstances make the consequence moderate (3).

The diesel flash point is $67^{\circ}$ which is much higher than the ambient temperature in addition to scare of ignition sources. These circumstances make the likelihood of fire low (2).

Based on the likelihood and consequence estimation, the 
risk level is estimated as 6 , which is considered low risk level with light green colour code, which requires continuous monitoring and improvement actions if any.

\subsubsection{Evaluate the Risk}

Based on the risk estimation, this risk is considered as low risk level which needs monitoring and improvement actions if any.

Recommended actions:

a) Periodical inspection for the diesel tank to ensure that there is no leakage or any sign of damage.

b) Prevent any hot work from being done near to the diesel tank except after getting hot work permit from the authorized person and implementing all required control measures.

c) Issue and apply restricted safety procedures and instructions for filling the tank with diesel by tankers e.g. grounding and bonding of both tank and tanker to avoid any electric spark because of static electricity.

These actions will help in keeping the risk level low and enhance its related safety standard.

\subsection{Risk Assessment for Uncovered Rotating Machinery and Equipment}

\subsubsection{Assessment Methodology}

This assessment will combine between qualitative and quantitative approaches for calculating the exposure time and estimate the likelihood. This risk assessment will follow the five steps to risk assessment model to identify the risk level and determine the required control measures. This assessment will focus mainly on the entanglement hazard of pumps as an example of the mechanical hazards of uncovered rotating machines and equipment in RO desalination plants.

\subsubsection{Identify the Hazard}

Pumps and energy recovery devices are main component of any RO desalination plant, in medium capacity $\left(10000 \mathrm{M}^{3 /}\right.$ day) plant there are almost 18 pumps, 6 high pressure pumps for supplying the membranes and 12 low pressure pumps for raw water and boosting, these pumps need daily inspection e.g. checking mechanical seal leakage, motor current, bearing housing temperature, noise, vibration and pressure. The inspection activities require direct contact between the technician and the pump while it is running. This situation could cause entanglement of the technician and the rotating shaft or coupling which could lead to severe injuries or fatalities especially it was noted that some pumps couplings are not covered or guarded.

\subsubsection{Estimating the Risk}

To estimate the likelihood of something wrong to happen, the exposure time should be calculated. After monitoring of inspection process, it was found that the average time for pump inspection is 10 minutes so the exposure time can be calculated as $10 \times 18=180$ minutes daily $=5400$ minutes monthly $=90 \mathrm{hrs} . /$ month.

From the previous calculations, it is clear that workers are exposed to the risk for significant time which makes the likelihood high (4).

Because of the high motor speed and power in addition to poor guarding of rotating elements, severity of consequences is estimated as high (4).

Based on the likelihood and consequence estimation, the risk level is estimated as 16 , which is considered high risk level with orange colour code.

\subsubsection{Evaluate the Risk}

As the risk rate is ranked by high, urgent action must be taken to protect employees and ensure they are safe while inspecting or maintaining pumps.

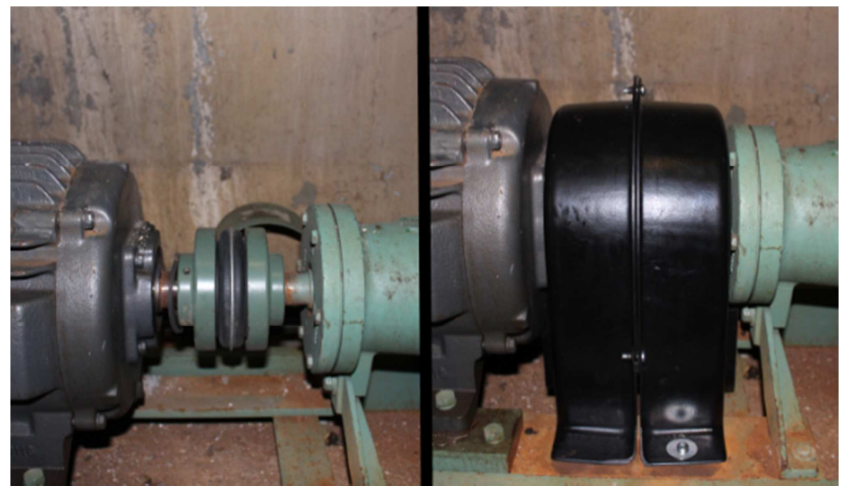

Figure 8. Guarding of rotating parts.

Recommended actions:

a) All rotating elements especially coupling, guards, chains, and flywheels should be guarded and periodically monitored to ensure it is effective. [13]

b) Reduce direct contact between workers and rotating elements as possible e.g. using the infrared thermometer to check the temperature from enough distance.

c) Provide safety instructions to all concerned workers to prevent them from wearing loose clothes, ties, watches or any item that could stuck with the rotating elements.

By applying these control measures the risk rank is expected to be reduced from the high level with orange colour code to low level with light green colour code, which requires continuous monitoring to ensure that the existing control measures still valid and effective, the required devices were delivered and that workers comply with the instruction. 


\begin{tabular}{|c|c|c|c|c|c|}
\hline $\begin{array}{c}\text { Severity } \\
\text { likelihood }\end{array}$ & $\begin{array}{c}\text { In significant } \\
(1)\end{array}$ & Minor (2) & Moderate (3) & Major (4) & $\begin{array}{c}\text { Catastrophic } \\
(5)\end{array}$ \\
\hline Very likely (5) & 5 & 10 & 15 & 20 & 25 \\
\hline Likely (4) & 4 & 8 & 12 & Before (16) & 20 \\
\hline Fairly likely (3) & 3 & 6 & 9 & 12 & 15 \\
\hline Unlikely (2) & 2 & 4 & 6 & 8 & 10 \\
\hline Very unlikely (1) & 1 & 2 & 3 & After (4) & 5 \\
\hline
\end{tabular}

Figure 9. Risk matrix uncovered rotating machines.

\subsection{Risk Assessment for Electrical Hazard}

\subsubsection{Assessment Methodology}

This assessment will be qualitative because of the difficulty to determine the actual time of working with electricity and numerically estimate the impact of electric shock on humans. The risk assessment will follow five steps to risk assessment model to estimate the likelihood and severity in order to calculate the risk rate.

\subsubsection{Identify the Hazard}

As electricity is the main power supply of the plant, all maintenance and even operation activities include dealing with electricity which introduces the risk of electric shock to workers especially because of poor maintained equipment, poor isolated electric cables or stored static electricity.

\subsubsection{Estimate the Risk}

As dealing with electricity is a repeated and usual activity, the likelihood of something wrong to happen is estimated as high (4).

Because of wide range of voltage (low, medium \& high) in addition to wet environment and based on the historical records for fatal accidents, the severity of consequence is estimated as very high (5).

Based on the likelihood and consequence estimation, the risk level is 20 , which is considered very high risk level with red colour code.

\subsubsection{Evaluate the Risk}

The risk rate is estimated as very high risk with red colour code, so any unsafe operation should be stopped and urgent action must be taken to protect workers and ensure their safety.

Recommended actions:

a) Regular and comprehensive check for all electrical connections, cables, plugs and outlets to ensure they are well insulated and do not introduce any risk in addition to change any deteriorated one.

b) Issue clear instructions for energy isolation before start working with electricity and strictly apply lock out/ tag out system. [14]

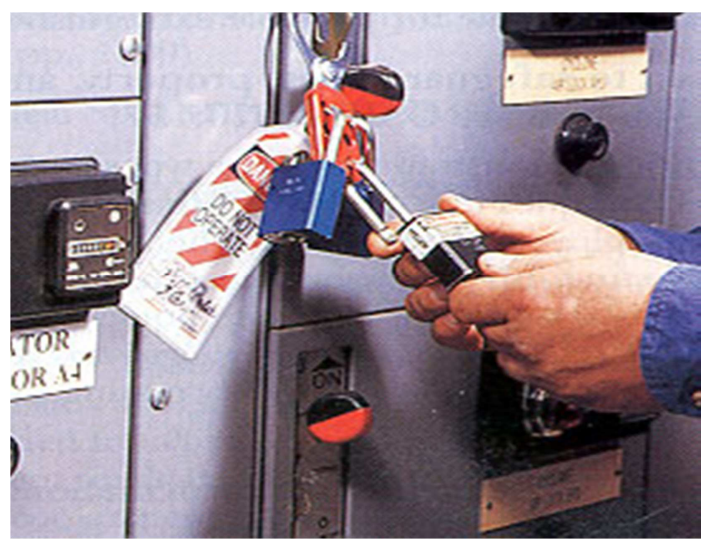

Figure 10. lock out/ tag out of electric panels.

c) Ensure that all workers are qualified enough and trained to deal with different ranges of voltage.

d) Provision of suitable equipment and tools e.g. insulated ladders, electric resistant gloves, safety shoes and voltage tester.

e) Relocate all electric cables that could contact with water in case of water leakage to be far from water.

f) Check all electric equipment to ensure it is effectively grounded.

g) Develop preventive maintenance and inspection procedures and schedules for all equipment to detect any sign of damage.

By implementing these control measures the risk level is supposed to be reduced from high level to low level with light green colour code which requires continuous monitoring, checking, inspection and improvement.

\begin{tabular}{|c|c|c|c|c|c|}
\hline $\begin{array}{c}\text { Severity } \\
\text { likelihood }\end{array}$ & $\begin{array}{c}\text { In significant } \\
(1)\end{array}$ & Minor (2) & Moderate (3) & Major (4) & $\begin{array}{c}\text { Catastrophic } \\
(5)\end{array}$ \\
\hline Very likely (5) & 5 & 10 & 15 & 20 & 25 \\
\hline Likely (4) & 4 & 8 & 12 & 16 & Before (20) \\
\hline Fairly likely (3) & 3 & 6 & 9 & 12 & 15 \\
\hline Unlikely (2) & 2 & 4 & After (6) & 8 & 10 \\
\hline Very unlikely (1) & 1 & 2 & 3 & 4 & 5 \\
\hline
\end{tabular}

Figure 11. Risk matrix for electric hazards. 


\subsection{Risk Assessment for High Pressurized Fluids}

\subsubsection{Assessment Methodology}

This assessment will be qualitative assessment depending on gathering information from manufacturer guidance, operation instructions and piping instrumentation diagram (PID) to identify pressurized lines, pressure values and safety aspects. The assessment will follow five steps to risk assessment model through estimating the likelihood and consequence in order to calculate the risk rate.

\subsubsection{Identify the Hazard}

Reverse osmosis process depends mainly on high pressure to separate saults and get pure water. Fluids under high pressure are considered serious hazard as it could cause severe injuries or fatalities in different ways such as releasing solid projectiles with high pressure in case of failure in the system e.g. releasing of pressure gage with 60 bar pressure, or high pressure injection e.g. high pressurized water release and penetrate the human body causing severe injuries. [15]

\subsubsection{Estimate the Hazard}

After reviewing the historical records for incidents, it is noted that failure in high pressurized lines is a repetitive near miss which could lead to real accident if not controlled. Although the chance of something go wrong is always there, it increases during maintenance activities. System failure could happen because of erosion of mechanical parts, bad assembly of components or exceeding the designed pressure. The likelihood is estimated as moderate (3).

The pressure at the discharge of high pressure pumps is 60-65 bars, in case of failure, the released fluids or solid parts with such pressure is able to cause fatalities or severe injuries if hit someone body so the severity is estimated as very high (5).

Based on the likelihood and consequence estimation, the risk level is estimated as 15 , which is considered high risk level with orange colour code.

\subsubsection{Evaluate the Risk}

As the risk rate is high, it needs urgent and immediate action to be controlled to protect the worker against such risk.

Recommended actions:

a) Carryout regular inspection of all high pressure pumps, energy recovery devices lines and vessels to detect any sign of damage and prevent potential failure.

b) Prepare for periodical inspection, replacing parts before its life time and preventive maintenance for pressurized systems by a competent staff.

c) Develop procurement criteria to include safety consideration as a main parameter of purchasing spare parts, tools and equipment and selecting vendors to avoid using of duplicated or low quality equipment.

d) Ensure that all used equipment and materials in pressurized systems is suitable to the water specification e.g. high salty water and that it meets the manufacturer standard.

e) Calibrate the pressure sensors and switches to ensure it is accurate and avoid increasing the pressure in the system more than the designed one.

f) Provide training to all concerned staff to ensure they are competent and familiar with the right way of assembly and disassembly of components and how to check the unit before starting.

g) Add maintenance of pressurized system to the list of tasks that must not be done except after getting work permit from the authorized person to ensure compliance with safety instructions such as isolating the power and discharging the stored pressure before opening the system.

By implementing these control measures the risk level is supposed to be reduced from high level to low level with light green colour code which requires continuous monitoring, checking, inspection and improvement.

\begin{tabular}{|c|c|c|c|c|c|}
\hline $\begin{array}{c}\text { Severity } \\
\text { likelihood }\end{array}$ & $\begin{array}{c}\text { In significant } \\
(1)\end{array}$ & Minor (2) & Moderate (3) & Major (4) & $\begin{array}{c}\text { Catastrophic } \\
(5)\end{array}$ \\
\hline Very likely (5) & 5 & 10 & 15 & 20 & 25 \\
\hline Likely (4) & 4 & 8 & 12 & 16 & 20 \\
\hline Fairly likely (3) & 3 & 6 & 9 & 12 & Before (15) \\
\hline Unlikely (2) & 2 & 4 & 6 & 8 & 10 \\
\hline Very unlikely (1) & 1 & 2 & 3 & 4 & After (5) \\
\hline
\end{tabular}

Figure 12. Risk matrix for pressurized fluid.

\subsection{Risk Assessment for Working in Immersed Water}

\subsubsection{Assessment Methodology}

This assessment will be qualitative because of the difficulty to calculate the real and accurate time of working under water. The assessment will follow five steps to risk assessment model in order to estimate the likelihood and consequence to calculate the risk level.

\subsubsection{Identify the Hazard}

Sea water is the feed water of RO plant; sea water intake point lies $14 \mathrm{~m}$ under the water surface $500 \mathrm{~m}$ far from the beach. Sometimes the intake line needs to be maintained, especially if it is blocked by suspended solids or aqua live organisms. Working in deep water exposes divers to death if 
stuck by water currents or vortices and cannot escape till the oxygen finished.

\subsubsection{Estimate the Risk}

After collecting information from the concerned staff about working under water, it is noted that this task needs to be done every long time and non-specified period so the likelihood of exposure to harm is estimated as low (2).

In case of something wrong occurred while working under water, the expected consequence is sever and mostly lead to fatalities, so the consequence is estimated as very high (5).

Based on the likelihood and consequence estimation, the risk level is estimated as 10 , which is considered moderate risk level with yellow colour code.

\subsubsection{Evaluate the Risk}

As the risk level is estimated as moderate with yellow colour code, action should be taken within a specified time scale.

Recommended actions:

a) Develop and strictly apply special work permit system to work under water and prevent anyone from starting such work without getting permit from the authorized person and after taking the required precautions.

b) Prevent lone working under water and prepare rescue plan in case of emergency.

c) Prevent carrying out any task under water while suction pumps are working to avoid vortices and water currents.

d) Provide right and suitable equipment for diving and rescue e.g. life lines in addition to training for divers and concerned workers.

By implementing these control measures the risk level is supposed to be reduced from moderate level to low level with light green colour code which requires continuous monitoring, checking, inspection and improvement.

\begin{tabular}{|c|c|c|c|c|c|}
\hline $\begin{array}{c}\text { Severity } \\
\text { likelihood }\end{array}$ & $\begin{array}{c}\text { In significant } \\
(1)\end{array}$ & Minor (2) & Moderate (3) & Major (4) & $\begin{array}{c}\text { Catastrophic } \\
(5)\end{array}$ \\
\hline Very likely (5) & 5 & 10 & 15 & 20 & 25 \\
\hline Likely (4) & 4 & 8 & 12 & 16 & 20 \\
\hline Fairly likely (3) & 3 & 6 & 9 & 12 & 15 \\
\hline Unlikely (2) & 2 & 4 & 6 & 8 & Before (10) \\
\hline Very unlikely (1) & 1 & 2 & 3 & After (4) & 5 \\
\hline
\end{tabular}

Figure 13. Risk matrix for working under water.

\section{Conclusion}

In the present work paper, the main hazards in RO desalination plants are identified and their risks are assessed. Standardized systematic approach for risk assessing is used. Both qualitative and quantitative models were included while assessing risks. The results are presented using numbers and colours in order to be easily prioritized.

Based on the assessment the main risks are prioritized as the following order: electric hazards, non-covered rotating machines, high pressurized fluid, and working at height, noise, working in confined space and in immersed water.

Although the previously mentioned are the most significant and most related hazards in RO desalination plants, there are many other hazards that are common in desalination plants as well as other industrial plants like slips, trips \& fall, Hazard of lifting operations, hazards of mobile equipment and ergonomic.

Also the controls are introduced to minimize these risks to an accepted level. This study can guide the concerned staff in RO desalination plants and help them to prevent accidents, protect workers and keep them safe.

\section{References}

[1] Barlow, m. And t. Clarke, blue gold: the battle against corporate theft of the world's water. 2017: routledge.
[2] Hernández-Sánchez, J. C., N. Boluda Botella, and J. L. Sánchez-Lizaso, The role of desalination in water management in southeast Spain. 2017.

[3] Karaghouli, a. And kazmerski, 1. Economic and technical analysis of reverse osmosis water desalınation plants using deep-3. 2 software, journal of environmental science \& engineering, vol. 1 1ssue 3a, p318, march 2012.

[4] ILO, occupational health and safety convention no 155,67 th 1lc session, artıcle 16, geneva, 1981.

[5] HSE, brief guide to controling risk in work place, health and safety executıve, merseyside, 2014.

[6] Hanada, v, accident prevention in desalınation plants, journal of desalination and water resources, plant operation managemen.

[7] Asem research task force on risk based inspection guidlıne, formed during 1986 and 1987.

[8] Donagi, water treatment plant operator hazard data sheet, international labour organisation, geneva, 2012.

[9] Shamseldin, a, chemical hazards in seawater desalination by the multistage-flash evaporation technique, journal of desalination and water resources, common fundamentals and unit operations in thermal desalination systems - vol. Iii.

[10] OSHA, fall protection system, 29 cfr 1910, occupational health and safety admistration, washington https://www.osha.gov/sltc/fallprotection/

[11] HSE, the control of noise at work regulation, the stationery office limited, UK, 2005. 
[12] HSE, controling noise at work $2^{\text {nd }}$ eddition, health safety executive, uk, 2005, page 58.

[13] Yates, a, safety professional's reference \& study guide, Taylor $\&$ Francis group, Florida, 2015, page 522.
[14] Brauer, 1, safety and health for engineers-second eddition, john wiley \& sons, new jersey, 2006, page 582.

[15] Manuele, f, advanced safety management focusing on serious injury pre, john wiley \& sons, new jersey, 2008, page 138. 